\title{
Clinical Characteristics and Prognostic Factors of Small Intestine Angiosarcoma: a Retrospective Clinical Analysis of 66 Cases
}

\author{
Rong Lia,b Ze-ying Ouyang ${ }^{\mathrm{a}}$ Jun-bo Xiao ${ }^{\mathrm{a}}$ Jian He $\mathrm{He}^{\mathrm{a}}$ Yan-wu Zhou \\ Gui-ying Zhang ${ }^{a}$ Qian Lia Huan Gua Ai-min Leng ${ }^{a}$ Ting Liu ${ }^{a}$ \\ a'Department of Gastroenterology, Xiangya Hospital of Central South University, Changsha, Hunan,

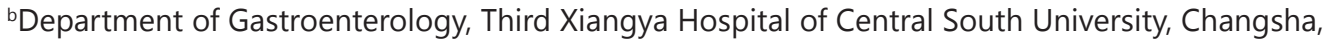 \\ Hunan, 'Department of Thoracic Surgery, Xiangya Hospital of Central South University, Changsha, \\ Hunan, China
}

\section{Key Words}

Small intestine angiosarcoma - Clinical characters - Prognosis - Gastrointestinal bleeding • Adjuvant therapy

\begin{abstract}
Background/Aims: Primary angiosarcoma of the small intestine is a rare neoplasia, and there are limited data from systematic analyses. The aim of this study is to describe the clinical and pathological characteristics in addition to the prognostic factors for this rare neoplasia. Methods: We retrospectively collected the clinical records and prognostic information of 66 patients with small intestine angiosarcoma reported between 1970 and 2017. We used the Chi-square test, the log-rank test, and Cox regression analyses to evaluate the data. Results: There were 66 patients diagnosed with small intestine angiosarcoma. The onset age ranged from 24-92 years old. There were 24 patients diagnosed before the year 2000, and 42 patients were diagnosed after 2000. The data indicated that 49 cases were diagnosed as primary disease, and the remaining 15 cases were secondary disease. The main clinical symptoms were nonspecific and included gastrointestinal (GI) bleeding and abdominal pain. Additionally, we found multi-center foci were one of the characteristics of this disease. Radiation-induced small intestine angiosarcoma (RSIA) is a special type of disease with a similar prognosis. This type was more frequent in females and decreased after the year 2000. We also found that GI bleeding was less common in RSIA cases. The log-rank test results revealed that old-age, poor differentiation, and GI bleeding were associated with worse prognosis. Surgical treatment showed a trend toward a prolonged survival time. However, the result was not statistically significant. Our results show treatment with adjuvant therapy improved prognosis. The multivariate Cox analysis demonstrated adjuvant therapy was an independent indicator of a favorable outcome in small intestine angiosarcoma patients. Conclusion: Pay attention to the unexplained gastrointestinal bleeding could lead to a faster diagnosis and control of small intestine angiosarcoma. Furthermore, treatments including adjuvant therapy can effectively improve the prognosis.




\section{Cellular Physiology \\ \begin{tabular}{l|l|l} 
and Biochemistry $10.1159 / 000485345$ & $\begin{array}{l}\text { C } 2017 \text { The Author(s). Published by S. Karger AG, Basel } \\
\text { www.karger.com/cpb }\end{array}$ \\
\hline
\end{tabular}}

Li et al.: Clinical Characteristics and Prognostic Factors of Small Intestine Angiosarcoma

\section{Introduction}

Angiosarcoma is a malignant tumor formed by the hyperproliferation of cells with vascular endothelial features. Angiosarcoma is a rare disease that represents 1-2\% of all sarcomas and frequently occurs in skin and subcutaneous tissues. This disease is less commonly observed in the following tissues: breast, liver, spleen, bone, retina, heart, ovaries, or the adrenal glands [1-6]. Disease with intestinal origin is extremely rare [7]. The first gastrointestinal angiosarcoma reported by Langhans $T$ in 1879 was found in the spleen [8]. There have been a limited number of cases reported since its initial discovery [9-13].

The specific etiology of angiosarcoma remains unclear. Although several factors including trauma, vinyl chloride, or radiation exposure have been implicated in disease development, the exposure to these agents is uncommon. The follow-up of patients treated with radiotherapy suggests the overall risk for post-radiation angiosarcoma development is $0.01 \%-0.3 \%[14]$.

The clinical manifestations of small intestine angiosarcoma are extremely variable and nonspecific [15-20]. Thus, histopathological detection is the most appropriate approach. However, pathology may be insufficient [20] for angiosarcomas showing high architectural and cytologic variability [21]. Moreover, even an experienced pathologist may have difficulty in diagnosing small intestine angiosarcoma. The ambiguous clinical manifestations and lack of biomarkers require laparotomy to confirm diagnosis and stabilize vital signs.

The resection of the bowel tumor is necessary but inadequate for the complete cure of intestinal angiosarcoma because the majority of cases are unresectable at diagnosis. Although chemotherapy is recommended for patients with angiosarcoma, there is currently no efficacious chemotherapeutic regimen. The common delayed diagnosis combined with an advanced stage lead to poor patient prognosis. The survival time reported in most cases range from 1 week to 3 years after resection of the foci [7, 22, 23].

Until now, only a small number of small intestine angiosarcoma cases have been reported sporadically. Unfortunately, not all cases have described the clinical outcomes in detail. Owing to its extremely low incidence, there is few statistical overview of small intestine angiosarcoma. No study has invistigated factors involved in the outcome of small intestine angiosarcoma systematically. Additionally, optimal management for small intestine angiosarcoma is poorly characterized. The distinctive manifestations of this tumor and the difficult diagnosis combined with high mortality make early discovery very important. In the present study, we have retrospectively analyzed 66 patients with small intestine angiosarcoma in order to identify potential factors involved in the occurrence of small intestine angiosarcoma and the potential indicators for the prognosis.

\section{Materials and Methods}

\section{Cases retrived}

We systematically searched in the online Web of Science, PubMed and Chinese databases (Wanfang, VIP and CNKI) (updated until March 7, 2017) with the restrictions of "English" language and "original article". The search terms used included "gastrointestinal", "small bowel", "small intestine" or "small intestinal" and "angiosarcoma", "haemangiosarcoma" or "angiomatous sarcoma". Published reviews were also included. We collected the demographic and clinicopathological information including year, country, gender, age, abdominal radiotherapy history, clinical manifestation, treatment modality (surgery or adjuvant therapy) and prognosis. Follow-up information was also included, and overall survival (OS) was defined as the period of time from the initial diagnosis of small bowel angiosarcoma to death from any cause or last follow-up.

\section{Cases selection}

In this study, cases were selected according to the following criteria: (1) case report of small intestine angiosarcoma with a clear diagnosis, clinical-pathological character and prognostic information; (2) study for overview of the small intestine angiosarcoma with summarized clinical information. However, 
unpublished cases, meeting abstracts, comments, letters and literature reviews were not included.

\section{Statistical analysis}

SPSS software (version 16.0, Chicago, IL) was used for statistical analyses in this study. All data were presented as mean \pm standard deviations (SD). Categorical variables were compared by $\chi 2$ or Fisher's exact test. Survival curves were done by Kaplan-Meier method (log-rank test). Univariate and multivariate analyses were performed by the Cox proportional hazard model. All tests were twotailed and a $P<0.05$ was considered to be statistically significant.

\section{Results}

The distribution of clinical characters in small intestine angiosarcoma

Owing to the fact that the small intestine angiosarcoma is a rare disease, there was only 1 case recorded in our hospital. After a comprehensive search for published case regarding small intestine angiosarcoma, we have totally consulted 59 references currently available, which included 66 cases information of small intestine angiosarcoma patient. The collection of clinical data was listed in Table 1.

A total of 46 male, 19 female were diagnosed with small intestine angiosarcoma worldwide, which suggested high male susceptibility. The average age of onset was 63.9-year old (ranged 24 - 92), 24 cases arised before 2000s and 42 cases happened in 2000-2017. 49 patients were proved to be primary intestinal angiosarcoma, the remaining 15 cases were metastatic disease. Angiosarcoma originated from skin or aorta showed a tendency of metastasis to small bowel. In these 66 cases, a total of 31 patients underwent emergency surgery because of intestinal perforation, obstruction or uncontrollable bleeding (data not shown).

42 reports provided detail pathological description, of which 17 cases were epithelioid, 10 cases were well differentiated, 11 cases were poorly differentiated and the remaining 4 cases were mixed. Due to the nonspecific clinical presentation, most small intestine angiosarcoma patients were clearly diagnosed by surgery. In addition, 10 patients had a clear diagnosis through the endoscopic
Table 1. The clinicopathological characteristics of 66 patients. NA. Not available

\begin{tabular}{|c|c|c|}
\hline Characteristics & Number of patients & $\%$ \\
\hline \multicolumn{3}{|l|}{ Gender } \\
\hline Male & 46 & 69.7 \\
\hline Female & 19 & 28.8 \\
\hline $\mathrm{NA}$ & 1 & \\
\hline \multicolumn{3}{|l|}{ Age } \\
\hline$\leq 65$ & 29 & 43.9 \\
\hline$>65$ & 35 & 53 \\
\hline $\mathrm{NA}$ & 2 & \\
\hline \multicolumn{3}{|l|}{ Race } \\
\hline Asian & 19 & 28.8 \\
\hline Non-Asian & 47 & 71.2 \\
\hline \multicolumn{3}{|l|}{ Year } \\
\hline$\leq 2000$ s & 24 & 36.4 \\
\hline$>2000 s$ & 42 & 63.6 \\
\hline \multicolumn{3}{|l|}{ Primary } \\
\hline Yes & 49 & 74.2 \\
\hline No & 15 & 22.7 \\
\hline NA & 2 & \\
\hline \multicolumn{3}{|l|}{ Size } \\
\hline$\leq 4 \mathrm{~cm}$ & 15 & 22.7 \\
\hline$>4 \mathrm{~cm}$ & 26 & 29.4 \\
\hline $\mathrm{NA}$ & 25 & \\
\hline \multicolumn{3}{|l|}{ Multiple center } \\
\hline Yes & 19 & 28.8 \\
\hline No & 35 & 53.0 \\
\hline $\mathrm{NA}$ & 12 & \\
\hline \multicolumn{3}{|c|}{ Pathological description } \\
\hline Epithelioid & 17 & 25.8 \\
\hline Well differentiated & 10 & 15.2 \\
\hline Poorly differentiated & 11 & 16.7 \\
\hline Mixed & 4 & 6.1 \\
\hline $\mathrm{NA}$ & 24 & \\
\hline \multicolumn{3}{|l|}{ Approach to diagnose } \\
\hline Endoscopic sample & 10 & 15.2 \\
\hline Surgic sample & 52 & 78.8 \\
\hline $\mathrm{NA}$ & 4 & \\
\hline \multicolumn{3}{|l|}{ Intervention } \\
\hline Surgery & 38 & 57.6 \\
\hline Adjuvant therapy & 16 & 24.2 \\
\hline None & 8 & 12.1 \\
\hline $\mathrm{NA}$ & 4 & \\
\hline \multicolumn{3}{|l|}{ GI bleeding } \\
\hline Yes & 41 & 56.1 \\
\hline No & 25 & 40.9 \\
\hline \multicolumn{3}{|c|}{ Abdominal radiotherapy } \\
\hline With & 17 & 25.8 \\
\hline Without & 49 & 74.2 \\
\hline
\end{tabular}


Table 2. Overview of fisrt manifestation in 66 cases

\begin{tabular}{lc}
\hline First manifestation & Number \\
\hline GI bleeding & 42 \\
Abdominal pain & 31 \\
Abdominal distension & 21 \\
Feeble and anemia & 20 \\
Nasuea and vomiting & 15 \\
Loss of weight & 7 \\
Short of breath & 5 \\
Loss of appetite & 4 \\
Fever & 3 \\
Drowsiness & 3 \\
Diarrhea & 2 \\
Hemoptysis & 2 \\
Peripheral edema & 1 \\
Hematuresis & 1 \\
Chest pain & 1 \\
Back pain & 1 \\
\hline
\end{tabular}

Table 3. The clinicalpathological characters of 66 patients with small intestine angiosarcoma grouped by radiation exposure

\begin{tabular}{|c|c|c|c|c|}
\hline Characteristics & $\mathrm{n}$ & With radiation exposure & Without radiation exposure & $p$ value \\
\hline Gender & & & & $<0.001$ \\
\hline Male & 46 & 6 & 40 & \\
\hline Female & 19 & 11 & 8 & \\
\hline Age & & & & 0.461 \\
\hline$\leq 65$ & 29 & 9 & 20 & \\
\hline$>65$ & 35 & 8 & 27 & \\
\hline Race & & & & 0.354 \\
\hline Asian & 19 & 3 & 16 & \\
\hline Non-Asian & 47 & 14 & 33 & \\
\hline Year & & & & $<0.001$ \\
\hline$\leq 2000$ & 24 & 11 & 13 & \\
\hline$>2000$ & 42 & 6 & 36 & \\
\hline Primary & & & & 0.013 \\
\hline Yes & 50 & 17 & 33 & \\
\hline No & 14 & 0 & 14 & \\
\hline Size & & & & 0.720 \\
\hline$\leq 4 \mathrm{~cm}$ & 15 & 3 & 12 & \\
\hline$>4 \mathrm{~cm}$ & 26 & 7 & 19 & \\
\hline Multiple center & & & & 0.206 \\
\hline Yes & 19 & 7 & 12 & \\
\hline No & 35 & 7 & 28 & \\
\hline Pathological description & & & & 0.891 \\
\hline Not Poorly differentiated & 31 & 12 & 19 & \\
\hline Poorly differentiated & 11 & 4 & 7 & \\
\hline GI bleeding & & & & 0.008 \\
\hline Yes & 41 & 6 & 35 & \\
\hline No & 25 & 11 & 14 & \\
\hline
\end{tabular}

biopsy, which showed an advantage in diagnosis with a minimally invasive approach. Among the 66 patients, 51 patients received surgery and/or adjuvant therapy, 7 patients gave up any further treatment because of unstable state or other sever comorbidities.

The main clinical symptoms for small intestine angiosarcoma were nonspecific, including gastrointestinal bleeding (GI bleeding), abdominal pain, abdominal distention, nausea and vomiting (Table 2). The extremely low incidence combined with the atypical clinical manifestations always result in missed or incorrect diagnoses. The diseases commonly misdiagnosed includes GIST, small intestinal lymphoma and intestinal adenocarcinoma, etc. According to our findings, 42 patients (85.7\%) received laparotomy with incorrect estimated preoperative diagnosis, even after a detailed preoperative examination and careful case discussion.

The characters of radioation-induced small intestine angiosarcoma (RSIA)

According to the potential cause of disease, angiosarcomas are classified into cutaneous angiosarcoma, primary-breast angiosarcoma, soft-tissue angiosarcoma, lymphoedemaassociated angiosarcoma and radiation-induced angiosarcoma [7]. As radiation is a proved efficacious treatment for some types of tumor including breast cancer, cervical cancer and uterine cancer, therefore, abdominal radiation therapy is widely used. Most of the documented cases of post-radiation angiosarcoma are reported in breast, skin and associated soft tissue [24]. Currently, post-radiation angiosarcoma has also been described in the small bowel, colon and omentum, even in the entire abdomen [25-27]. In the present study, we have retrospectively detected 17 cases of RSIA among the 66 intestinal angiosarcomas. Radiation therapy was initially employed in the adjuvant setting in these cases. Radiation doses ranged from 8 (single fraction) -91.2 Gy with a median of $60 \mathrm{~Gy}$. Consistent with the features of reported radiation ralated angiosarcoma [25, 27], the average interval between radiation exposure and presentation of RSIA was 10 years, ranged from 3 to 20 years. 
Fig. 1. (A, B) Race and gender did not influence OS of small intestine angiosarcoma. (C) Age did not impact the OS when cut off set at 65-year old significantly, however, (D) patients younger than 50-year old showed a longer survival time than those older than 50-year old.

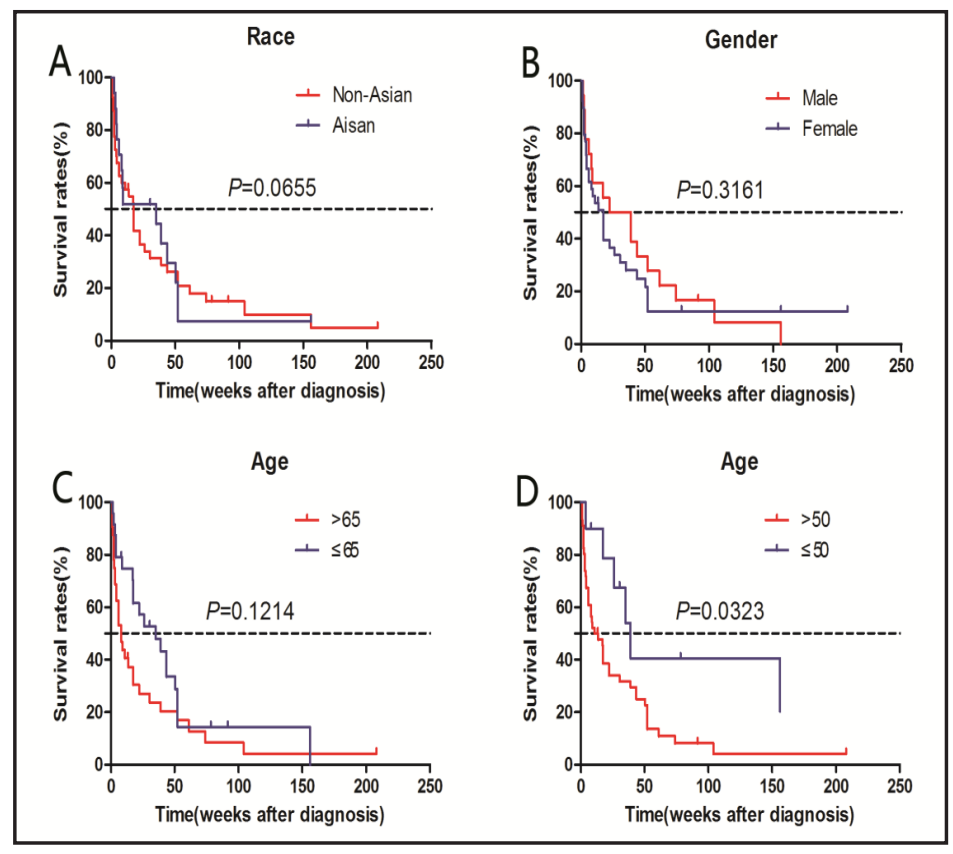

Herein, we have analyzed the association between clinical characters and the occurrence of RSIA. As displayed in Table 3, classification by age and race did not reveal any particular distribution, while the female were more inclined to develop small intestinal angiosarcoma than male $(11 / 19$ vs. $6 / 46, P<0.001)$. Moreover, more cases of small intestinal angiosarcoma were found and reported in 1970-2000 than in 2000-2017 (11/24 vs. $6 / 42, P=0.004)$. Intriguingly, the GI bleeding was much less frequent in the patients with radiotherapy history than those without $(6 / 17$ vs. $35 / 49, P=0.028)$.

\section{The factors influencing the OS of small intestine angiosarcoma}

The prognosis was much worse for patients with small intestine angiosarcomas than those with tumors of any other site, and over one-year survival time is extremely rare [28]. Most patients did not survive the post-operative period or died within a few months after diagnosis. In the present study, we have also concluded and summarized outcome of 58 patients with intact follow-up information. The prognosis of small intestine angiosarcoma was very poor for that the average survival time was about 30 weeks, with a range from 1 week to 208 weeks after clear diagnosis. Moreover, uncontrollable GI bleeding or acute respiratory failure was common direct cause of death.

Kaplan-Meier and log-rank test method were used to analyze the effects of race, gender, age, clinical manifestation, and type of treatment on OS. As displayed in Fig. 1, race, gender and age reached no significance with survival time. However, when the cutoff value of age was decreased to 50 year-old, a better prognosis was found in patients in less than 50 -year old group, 3-time longer than in the older group with a median survival time of 39 weeks. The origin and size of angiosarcoma or radiotherapy history did not impact the outcome (Fig. 2). However, poor differentiation was associated with shorter OS. In addition, GI bleeding has a significantly negative impact on OS, the median survival time was 8.5 weeks in the patients with GI bleeding, compared to 50.4 weeks in the patients without (Fig. 3A, 3B).

Timing and type of the intervention to the process have been demonstrated to predict the prognosis [29]. In the 58 cases with intact follow-up information, 38 patients received surgery alone, 13 patients received surgery and adjuvant therapy (radiotherapy or chemotherapy) and 7 gave up any treatment. The intervention helped to acquire longer survival time. The average OS of small intestine angiosarcoma was 17.22 weeks in surgery group and 84.65 weeks in adjuvant therapy group when compared to 2.85 weeks in those 
Fig. 2. $(\mathrm{A}, \mathrm{B})$ The origin or size of tumor played an inconspicuous role in OS of small intestine angiosarcoma. (C) Radiation-induced intestine angiosarcoma displayed a similar outcome to those without radiation exposure. (D) The prognosis was not improved after 2000s.
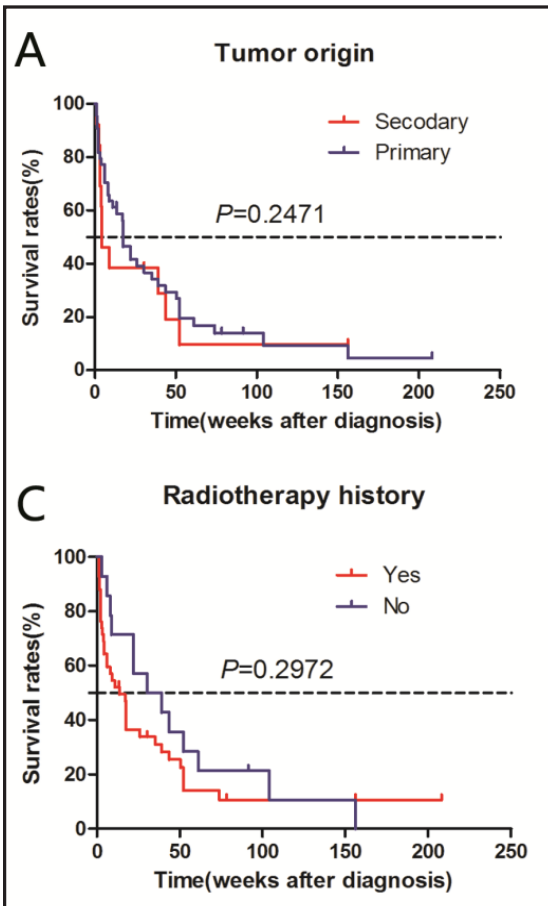
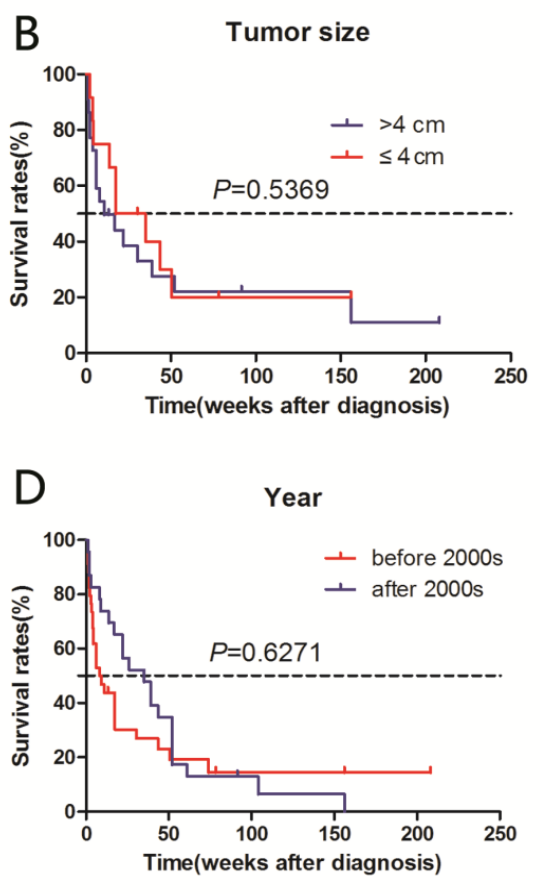

Fig. 3. (A) Poor differentiation and (B) GI bleeding of small intestine angiosarcoma aggravated a poorer outcome. (C, D) Surgery did not improve the prognosis, but adjuvant chemotherapy did.
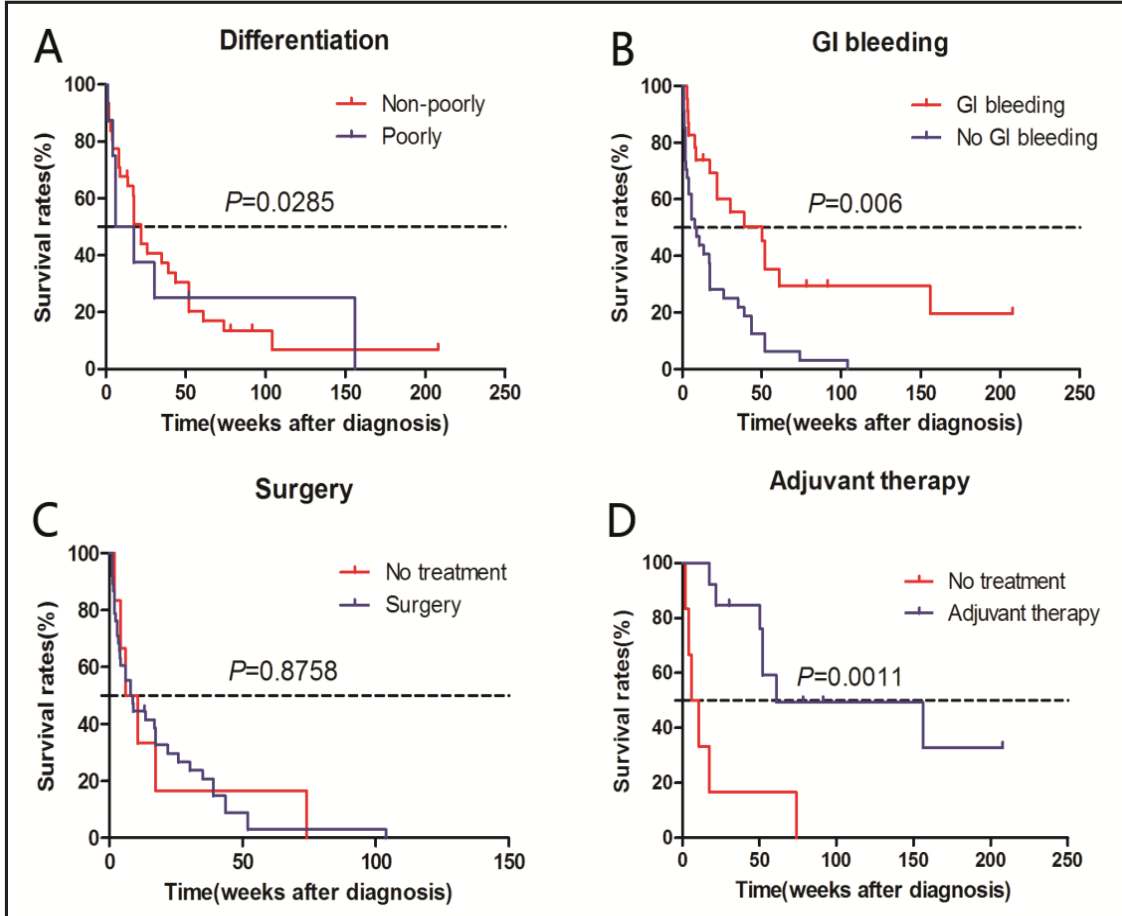

without any intervention. However, surgery did not prolong OS significantly, according to analysis of the present available data (Fig. 3C, 3D).

\section{The factors indicating the prognosis of small intestine angiosarcoma}

Based on the present study, it was worthwhile to consider major determinants in the prognosis of small intestine angiosarcoma using the Cox proportional hazards regression model. As univariate analyses (Table 4) displayed that the gender, age, race or radiotherapy history did not influence OS $(P>0.05)$. GI bleeding indicated a much worse outcome for 


\section{Cellular Physiology Cell Physiol Biochem 2017;44:817-827 \\ \begin{tabular}{l|l} 
and Biochemistry Publisher 10.1159/000485345 & $\begin{array}{l}\text { (c) } 2017 \text { The Author(s). Published by S. Karger AG, Basel } \\
\text { www.karger.com/cpb }\end{array}$
\end{tabular} \\ Li et al.: Clinical Characteristics and Prognostic Factors of Small Intestine Angiosarcoma}

the small intestine angiosarcoma patients, while adjuvant therapy did decrease the risk of death greatly. Surprisingly, surgery, however, did not benefit patient survival. Moreover, the multivariate Cox analysis demonstrated that the adjuvant therapy was an independent indicator for favorable prognosis of small intestine angiosarcoma (Table 5).

\section{Discussion}

Although the small intestine constitutes $75 \%$ of the GI tract, primary small bowel malignant tumors are rare, and the incidence is approximately 1 per 100,000 [30]. Furthermore, small intestine angiosarcoma is an extremely unusual disease and accounts for approximately $2 \%$ soft tissue sarcomas [7].

Secondary neoplastic involvement of the small bowel is more frequent than primary small bowel neoplasm and occurs by hematogenous spread, direct invasion, or intraperitoneal seeding [31]. However, our findings indicate the rate of metastasis to the intestine was unusual in small intestine angiosarcoma, and this finding is consistent with previous results $[20,32]$. This abnormal phenomenon may be attributed to the highly malignant nature of angiosarcoma, which is associated with severe clinical manifestations before spreading from the primary tumor to the intestine.

The common initial symptoms can vary from anemia to gastrointestinal hemorrhage. This variability in clinical presentation makes it difficult to quickly obtain an accurate diagnosis. Although endoscopic biopsies seem helpful [33, 34], surgery remains the only effective diagnostic method for this particular disease. Moreover, careful follow-up is helpful especially when there is recurrent abdominal pain or intestinal obstruction presented in the setting of unexplained anemia or gastrointestinal hemorrhage. For example, a patient initially presented with anemia and melena had been misdiagnosed with an aorto-intestinal fistula due to his aortic dilation history. However, the intestinal bleeding did not arrest after stent placement and aortic replacement. An exploratory laparotomy helped to clarify the diagnosis [17]. Another case of intestinal angiosarcoma masqueraded as an appendiceal abscess. The vigilance of unexplained microcytic anemia helped to clarify the prognosis and reiterated the variable nature of the tumor manifestation [35].

The patients and tumor characteristics in our series are consistent with previous studies of angiosarcoma $[22,23]$. Small intestine angiosarcoma showed a strong male predominance, with a male to female ratio of 2.42:1 [36]. This finding could be attributed to the increased chance of exposure to industrial chemicals for men. However, there were only 3 patients in the present study with a clear prior history of exposure to definitive causative chemical agents. The recent increased awareness of prevention and protection has decreased the chances of being exposed to these carcinogens. The morbidity of this disease has increased gradually, which indicates the majority of small intestine angiosarcomas are not related to these factors.
Table 4. Univariate cox analysis of survival in 58 patients with intestine angiosarcoma

\begin{tabular}{lll}
\hline Characteristics & HR(95\%CI) & $p$ Value \\
\hline Year (after 2000/before 2000) & $1.251(0.708-2.213)$ & 0.441 \\
Gender (female/male) & $0.847(0.467-1.535)$ & 0.584 \\
Age (year) ( $>50 / \leq 50)$ & $1.676(0.748-3.758)$ & 0.210 \\
Racial (Non-Asian/Asian) & $1.083(0.580-2.019)$ & 0.803 \\
Radiotherapy history (With/Without) & $1.391(0.733-2.641)$ & 0.312 \\
Differentiation (poorly/not poorly) & $1.104(0.472-2.581)$ & 0.819 \\
Tumor size (>4cm/s4cm) & $1.288(0.572-2.903)$ & 0.542 \\
Multiple foci (yes/no) & $0.836(0.434-1.607)$ & 0.590 \\
GI bleeding (no/yes) & $0.361(0.194-0.672)$ & 0.001 \\
Secondary / Primary & $1.182(0.602-2.320)$ & 0.628 \\
Metastasis (yes/no) & $0.845(0.198-3.600)$ & 0.819 \\
Intervention & & \\
Surgery / None & $1.137(0.473-2.735)$ & 0.774 \\
Adjuvant therapy / None & $0.176(0.056-0.555)$ & 0.003 \\
\hline
\end{tabular}

Table 5. Multivariate cox analysis of survival in 58

\begin{tabular}{lcc}
\hline Characteristics & HR $(95 \% \mathrm{CI})$ & $p$ Value \\
\hline GI bleeding (no/yes) & $1.178(0.543-2.555)$ & 0.679 \\
Intervention & & 0.002 \\
Surgery (yes/no) & $1.097(0.448-2.689)$ & 0.839 \\
Adjuvant therapy (yes/no) & $0.149(0.036-0.605)$ & 0.008 \\
\hline
\end{tabular}
patients with small intestine angiosarcoma 
Although the precise predisposing factors of small intestine angiosarcoma remain unclear, radiotherapy has been implicated in the pathogenesis of this tumor. Recent advances in radiotherapy technology have increased the number of patients cured of their primary malignancies and has also increased the prevalence of radiotherapy associated small intestine angiosarcoma [37]. Our findings suggest radiotherapy was responsible for $25.7 \%$ of all small intestine angiosarcoma cases. RSIA was more likely to occur in women, and the incidence rate for females was 3-fold higher than the rate for men. These findings are consistent with the increased use of radiotherapy for particular female genital tract malignancies. The clinical manifestations of RSIA at presentation were typically related to perforated viscus or intestinal obstruction. An unexpected frequent clinical manifestation of small intestine angiosarcoma is GI bleeding. However, this sign was not common in RSIA cases. These results might be explained by the findings that abdominal radiotherapy was often accompanied by fibrosis and reduced blood flow. Interestingly, the rate of RSIA decreased after the year 2000, while the overall incidence of small intestine angiosarcoma increased. The decreased RSIA rate indicates that proper organ protection during radiotherapy can effectively prevent RSIA. The terminal ileum was the most common location of RSIA [38].

There is currently no systemic analysis of small intestine angiosarcoma in the literature. This is the first study to analyze factors affecting the prognosis of small intestine angiosarcoma. Our results showed that there was no difference in OS between races. Moreover, age was neither a factor to impact the prognosis, when the cut off set at 65-year old. Small intestine angiosarcoma was the disease with a tendency to bleeding. Its associated bleeding episode was always multicenter and difficult to control, which was usually responsible for emergency laparotomy or even death [39]. Moreover, GI bleeding has also been demonstrated to be an indicator for an unfavorable prognosis in the present study. It was shown to shorten the average survival time from 50.4 weeks to 8.5 weeks. Therefore, it might be beneficial to step up vigilance on GI bleeding. RSIA displayed a relatively clear pathogenesis and had a longer average OS (34.7 weeks) compared to those without radiotherapy history (13.6 weeks). However, the difference was not statistically significant even if analyzed as a sub-group. The reduced chance of GI bleeding in RSIA might be the possible explanation.

The incidence of malignant intestine tumors has increased worldwide, and the management of abdominal oncologic emergencies has become more clinically important. Surgery has been the treatment of choice for small intestine angiosarcoma and was considered the only therapeutic modality with curative potential [40]. Our analysis showed that surgical resection did not play an important role in patient outcome. This was mainly due to the substantial individual differences within the group, and the survival time in the surgery group ranged from 1 week to 208 weeks. Conversely, surgery was always used and complete resection was often not possible due to the infiltrative nature of the tumor. Thus, conclusions regarding the limited therapeutic merits of tumor resection must be offered with considerable circumspection. Cumulatively, the results indicate a large trial and further investigations are warranted to validate our conclusion.

Though little systematic evidence supported that chemotherapy was efficacious for intestine angiosarcoma, palliative chemotherapy still could be an option. Studies of soft tissue sarcoma suggested that adjuvant therapy would help to prolong progression-free survival even in metastatic angiosarcoma [41]. Moreover, the only currently available intestine angiosarcoma cases treated with chemotherapy regimen have shown favorable and promising efficacy [42]. Our present study has also demonstrated that adjuvant therapy helped improve the outcome of small intestine angiosarcoma significantly, which accorded with the findings in advanced hepatic angiosarcoma [36]. However, it is worth noting that most adjuvant therapy protocols were based on studies of cutaneous angiosarcoma and generally empiric, as randomized clinical studies on intestinal angiosarcomas were lacking due to their rarity. Before credible evidence-based basis derived from further large-scale trails, Taxol and thalidomide could be considered because of their anti-angiogenic properties $[18,43]$. Docetaxel, ifosfamide, or recombinant human interleukin-2 has also shown their potential therapeutic efficacy $[44,45]$.

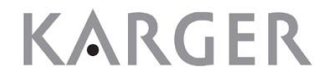




\section{Cellular Physiology

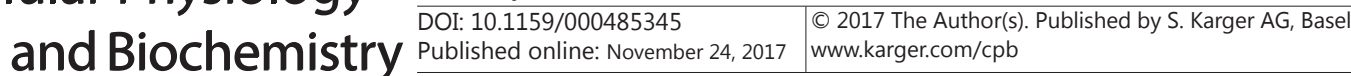

The present study has taken the first step to systematically analyze the clinicopathological features and potential prognostic determinants in small intestine angiosarcoma. We found that radiation associated small intestine angiosarcoma might be a special type, but with a similar outcome. Recurrent GI bleeding is one of the most common tumor manifestations and is both a direct cause of death and a predictor of worse outcome. Moreover, the findings in the present study suggest that treatment with adjuvant therapy significantly improves patient prognosis. However, due to the rare incidence and limited number of cases a statistical bias is inevitable. Therefore, a more comprehensive and a larger scale trial is warranted. We anticipate that our study will be a useful reference for developing new treatments in patients with small intestine angiosarcoma.

\section{Acknowledgements}

The authors thank Prof. Jian-gang Liu and Prof. You-e He for their helpful comments and suggestions of manuscript modification.

\section{Disclosure Statement}

None.

\section{References}

1 Locker GY, Doroshow JH, Zwelling LA, Chabner BA: The clinical features of hepatic angiosarcoma: a report of four cases and a review of the English literature. Medicine (Baltimore) 1979;58:48-64.

$\longrightarrow 2$ Pereira TC, Vi LH, Prichard JW, Sturgis CD: Pathologic quiz case. Elderly man with bright red blood per rectum. Pathologic diagnosis: metastatic hepatic epithelioid angiosarcoma. Arch Pathol Lab Med 2001;125:968-970.

- 3 Popper H, Thomas LB, Telles NC, Falk H, Selikoff IJ: Development of hepatic angiosarcoma in man induced by vinyl chloride, thorotrast, and arsenic. Comparison with cases of unknown etiology. Am J Pathol 1978;92:349-376.

4 Smith VC, Eisenberg BL, McDonald EC: Primary splenic angiosarcoma. Case report and literature review. Cancer 1985;55:1625-1627.

5 Liu Q, Ouyang R, Chen P, Zhou R: A case report of retiform hemangioendothelioma as pleural nodules with literature review. Diagn Pathol 2015;10:194.

6 Tang K, Shang QL, Zhou QC, Zhou JW, She XL, Zhang M: Primary cardiac angiosarcoma with spontaneous ruptures of the right atrium and right coronary artery. Echocardiography 2013;30:E156-160.

7 Young RJ, Brown NJ, Reed MW, Hughes D, Woll PJ: Angiosarcoma. Lancet Oncol 2010;11:983-991.

8 Langhans T: Pulsating cavernous neoplasm of the spleen with metastatic nodules to the liver. Vichows Arch Pathol Anat 1879;75:18.

-9 Ruffolo C, Angriman I, Montesco MC, Scarpa M, Polese L, Barollo M, Pagano D, D’Amico DF: Unusual cause of small bowel perforation: metastasis of a subcutaneous angiosarcoma of the head. Int J Colorectal Dis 2005;20:551-552.

10 Schmid E, Port SJ, Carroll RM, Friedman NB: Primary metastasizing aortic endothelioma. Cancer 1984;54:1407-1411.

11 Kunkel D, Duval JL, Bouchiat C, Talard P, Dubayle P, Carloz E, Flandrin P: [Angiosarcoma of the aorta revealed by an intestinal metastasis]. Gastroenterol Clin Biol 1993;17:139-141.

12 Bandorski D, Arps H, Jaspersen D, Diehl KL: Severe intestinal bleeding caused by intestinal metastases of a primary angiosarcome of the thyroid gland. Z Gastroenterol 2002;40:811-814.

13 Hsu JT, Lin CY, Wu TJ, Chen HM, Hwang TL, Jan YY: Splenic angiosarcoma metastasis to small bowel presented with gastrointestinal bleeding. World J Gastroenterol 2005;11:6560-6562. 


\section{Cellular Physiology Cell Physiol Biochem 2017;44:817-827 \begin{tabular}{l|l|l|l|} 
DOI: 10.1159/000485345 & O 2017 The Author(s). Published by S. Karger AG, Basel \\
wwww.karger.com/cpb
\end{tabular}

- 14 Mark RJ, Poen J, Tran LM, Fu YS, Selch MT, Parker RG: Postirradiation sarcomas. A single-institution study and review of the literature. Cancer 1994;73:2653-2662.

15 Delvaux V, Sciot R, Neuville B, Moerman P, Peeters M, Filez L, Van Beckevoort D, Ectors N, Geboes K: Multifocal epithelioid angiosarcoma of the small intestine. Virchows Arch 2000;437:90-94.

16 Saad A, Cappell MS, Amin M: Endoscopic findings with GI angiosarcoma correspond with the propensity of these vascular tumors to cause GI bleeding: two case reports and review of the literature. Dig Dis Sci 2013;58:1797-1801.

17 Zacarias Fohrding L, Macher A, Braunstein S, Knoefel WT, Topp SA: Small intestine bleeding due to multifocal angiosarcoma. World J Gastroenterol 2012;18:6494-6500.

-18 Allison KH, Yoder BJ, Bronner MP, Goldblum JR, Rubin BP: Angiosarcoma involving the gastrointestinal tract: a series of primary and metastatic cases. Am J Surg Pathol 2004;28:298-307.

$\checkmark 19$ Su CC, Jin YT, Chien CH, Yu CY, Lin PW: Postirradiation angiosarcoma of the terminal ileum. Zhonghua Yi Xue Za Zhi (Taipei) 1991;48:147-152.

20 Uchihara T, Imamura Y, Iwagami S, Kajihara I, Kanemaru H, Karashima R, Ida S, Ishimoto T, Baba Y, Sakamoto Y, Miyamoto Y, Yoshida N, Watanabe M, Iyama K, Ihn H, Baba H: Small bowel perforation due to indistinguishable metastasis of angiosarcoma: case report and brief literature review. Surg Case Rep 2016;2:42.

21 Al Ali J, Ko HH, Owen D, Steinbrecher UP: Epithelioid angiosarcoma of the small bowel. Gastrointest Endosc 2006;64:1018-1021.

-22 Lahat G, Dhuka AR, Hallevi H, Xiao L, Zou C, Smith KD, Phung TL, Pollock RE, Benjamin R, Hunt KK, Lazar AJ, Lev D: Angiosarcoma: clinical and molecular insights. Ann Surg 2010;251:1098-1106.

-23 Fayette J, Martin E, Piperno-Neumann S, Le Cesne A, Robert C, Bonvalot S, Ranchere D, Pouillart P, Coindre JM, Blay JY: Angiosarcomas, a heterogeneous group of sarcomas with specific behavior depending on primary site: a retrospective study of 161 cases. Ann Oncol 2007;18:2030-2036. Koplin SA, Twohig MH, Lund DP, Hafez GR: Omental lipoblastoma. Pathol Res Pract 2008;204:277-281. Nanus DM, Kelsen D, Clark DG: Radiation-induced angiosarcoma. Cancer 1987;60:777-779. Wolov RB, Sato N, Azumi N, Lack EE: Intra-abdominal "angiosarcomatosis" report of two cases after pelvic irradiation. Cancer 1991;67:2275-2279.

27 Westenberg AH, Wiggers T, Henzen-Logmans SC, Verweij J, Meerwaldt JA, van Geel AN: Post-irradiation angiosarcoma of the greater omentum. Eur J Surg Oncol 1989;15:175-178.

28 Chami TN, Ratner LE, Henneberry J, Smith DP, Hill G, Katz PO: Angiosarcoma of the small intestine: a case report and literature review. Am J Gastroenterol 1994;89:797-800.

29 Turan M, Karadayi K, Duman M, Ozer H, Arici S, Yildirir C, Kocak O, Sen M: Small bowel tumors in emergency surgery. Ulus Travma Acil Cerrahi Derg 2010;16:327-333.

-30 Barclay TH, Schapira DV: Malignant tumors of the small intestine. Cancer 1983;51:878-881.

31 Gill SS, Heuman DM, Mihas AA: Small intestinal neoplasms. J Clin Gastroenterol 2001;33:267-282.

32 Ni Q, Shang D, Peng H, Roy M, Liang G, Bi W, Gao X: Primary angiosarcoma of the small intestine with metastasis to the liver: a case report and review of the literature. World J Surg Oncol 2013;11:242.

-33 Knop FK, Hansen MB, Meisner S: Small-bowel hemangiosarcoma and capsule endoscopy. Endoscopy 2003;35:637.

34 Maeyashiki C, Nagata N, Uemura N: Angiosarcoma involving solid organs and the gastrointestinal tract with life-threatening bleeding. Case Rep Gastroenterol 2012;6:772-777.

-35 Liu DS, Smith H, Lee MM, Djeric M: Small intestinal angiosarcoma masquerading as an appendiceal abscess. Ann R Coll Surg Engl 2013;95:e22-24.

36 Kim HR, Rha SY, Cheon SH, Roh JK, Park YN, Yoo NC: Clinical features and treatment outcomes of advanced stage primary hepatic angiosarcoma. Ann Oncol 2009;20:780-787.

37 Garg G, Massad LS, Pourabolghasem S, Zhou G, Powell MA, Thaker PH, Hagemann AR, WilkinsonRyan I, Mutch DG: Intestinal perforation in gynecologic oncology: do all patients benefit from surgical management? Gynecol Oncol 2013;129:538-543.

-38 Chen KT, Hoffman KD, Hendricks EJ: Angiosarcoma following therapeutic irradiation. Cancer 1979;44:2044-2048.

-39 Grewal JS, Daniel AR, Carson EJ, Catanzaro AT, Shehab TM, Tworek JA: Rapidly progressive metastatic multicentric epithelioid angiosarcoma of the small bowel: a case report and a review of literature. Int J Colorectal Dis 2008;23:745-756. 


\section{Cellular Physiology Cell Physiol Biochem 2017;44:817-827

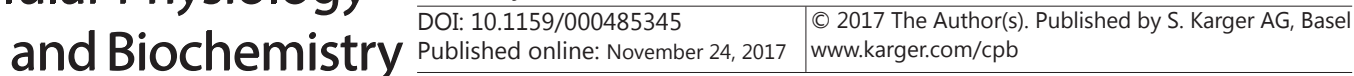

40 Policarpio-Nicolas ML, Nicolas MM, Keh P, Laskin WB: Postradiation angiosarcoma of the small intestine: a case report and review of literature. Ann Diagn Pathol 2006;10:301-305.

41 Krikelis D, Judson I: Role of chemotherapy in the management of soft tissue sarcomas. Expert Rev Anticancer Ther 2010;10:249-260.

-42 Ray-Coquard I, Italiano A, Bompas E, Le Cesne A, Robin YM, Chevreau C, Bay JO, Bousquet G, PipernoNeumann S, Isambert N, Lemaitre L, Fournier C, Gauthier E, Collard O, Cupissol D, Clisant S, Blay JY, Penel N, French Sarcoma G: Sorafenib for patients with advanced angiosarcoma: a phase II Trial from the French Sarcoma Group (GSF/GETO). Oncologist 2012;17:260-266.

-43 Fraiman G, Ganti AK, Potti A, Mehdi S: Angiosarcoma of the small intestine: a possible role for thalidomide? Med Oncol 2003;20:397-402.

44 Stacchiotti S, Palassini E, Sanfilippo R, Vincenzi B, Arena MG, Bochicchio AM, De Rosa P, Nuzzo A, Turano S, Morosi C, Dei Tos AP, Pilotti S, Casali PG: Gemcitabine in advanced angiosarcoma: a retrospective case series analysis from the Italian Rare Cancer Network. Ann Oncol 2012;23:501-508.

45 Penel N, Van Glabbeke M, Marreaud S, Ouali M, Blay JY, Hohenberger P: Testing new regimens in patients with advanced soft tissue sarcoma: analysis of publications from the last 10 years. Ann Oncol 2011;22:1266-1272. 